\title{
ARTICLE
}

Received 16 Mar 2015 | Accepted 21 May 2015 | Published 20 Jul 2015

DOI: $10.1038 /$ ncomms 8584

OPEN

\section{Solubility design leading to high figure of merit in low-cost $\mathrm{Ce}-\mathrm{CoSb}_{3}$ skutterudites}

Yinglu Tang ${ }^{1,2}$, Riley Hanus ${ }^{2}$, Sinn-wen Chen $^{3} \&$ G. Jeffrey Snyder ${ }^{2}$

$\mathrm{CoSb}_{3}$-based filled skutterudite has emerged as one of the most viable candidates for thermoelectric applications in automotive industry. However, the scale-up commercialization of such materials is still a challenge due to the scarcity and cost of constituent elements. Here we study $\mathrm{Ce}$, the most earth abundant and low-cost rare earth element as a single-filling element and demonstrate that, by solubility design using a phase diagram approach, the filling fraction limit ( $\mathrm{FFL}) x$ in $\mathrm{Ce}_{x} \mathrm{Co}_{4} \mathrm{Sb}_{12}$ can be increased more than twice the amount reported previously $(x=0.09)$. This ultra-high FFL $(x=0.20)$ enables the optimization of carrier concentration such that no additional filling elements are needed to produce a state of the art $n$-type skutterudite material with a $z T$ value of 1.3 at $850 \mathrm{~K}$ before nano-structuring. The earth abundance and low cost of Ce would potentially facilitate a widespread application of skutterudites.

\footnotetext{
${ }^{1}$ Department of Applied Physics and Materials Science, California Institute of Technology, Pasadena, California 91125, USA. ${ }^{2}$ Department of Materials Science and Engineering, Northwestern University, Evanston, Illinois 60208, USA. ${ }^{3}$ Department of Chemical Engineering, National Tsing Hua University, \#101, Sec.2, Kuang-Fu Rd., Hsin-Chu 300, Taiwan. Correspondence and requests for materials should be addressed to G.J.S. (email: jeff.snyder@northwestern.edu).
} 
$\mathrm{T}$ he world energy consumption has been increasing steadily and much effort is required worldwide to meet the challenging demand. Thermoelectric materials, which can convert waste heat into electricity, may help to reduce fossil fuel consumption and augment energy efficiency, and thus play an important role in the solution for the world's energy dilemma. The thermoelectric performance of materials is characterized by the dimensionless figure of merit, $z T=S^{2} \sigma T / \kappa$, where $S$ is the Seebeck coefficient, $\sigma$ is the electrical conductivity, $T$ is the absolute temperature and $\kappa$ is the total thermal conductivity.

Among various kinds of state-of-the-art thermoelectric materials, filled skutterudites distinguish themselves by allowing low thermal conductivity like a glass and high electrical conductivity like a crystal at the same time, which make them excellent thermoelectric materials according to the 'phonon glass electron crystal' concept ${ }^{1}$. Moreover, the intermediate temperature range $\left(400-600^{\circ} \mathrm{C}\right)$ at which filled skutterudites have optimum thermoelectric performance makes this material a perfect candidate for automotive industry applications. Because of the heavy mass and small radius of the $\mathrm{Yb}$ atom compared with other fillers, $\mathrm{Yb}-\mathrm{CoSb}_{3}$ skutterudites have excellent thermoelectric properties $\left(z T=1.3\right.$ at $850 \mathrm{~K}$ for $\left.\mathrm{Yb}_{0.3} \mathrm{Co}_{4} \mathrm{Sb}_{12}\right)$ ref. 2 and have been selected by many automotive companies, such as General Motors, Ford and BMW, as the most promising thermoelectric material for waste heat recovery ${ }^{3}$. However, the deficiency of the rare earth element $\mathrm{Yb}$ (abundance in earth's crust 3 p.p.m.) could potentially be a problem in large-scale commercialization. Unlike $\mathrm{Yb}, \mathrm{Ce}$ is the most earth abundant element of the rare earths (abundance in earth's crust 68 p.p.m.). ${ }^{4}$. In fact, there are huge resources of cerium because it is more abundant than other commonly known elements such as lead (14 p.p.m.), tin (2 p.p.m.), silver (0.07 p.p.m.), tellurium (0.005 p.p.m.) and gold (0.001 p.p.m.). The price of cerium is also more competitive compared with ytterbium. For large quantities, the difference is a factor of 10 (Ce; $\$ 5,000$ per ton, Yb; $\$ 50,000$ per ton ${ }^{5}$ ), placing Ce in the category of other commonly used elements such as $\mathrm{Ni}$.

With its larger earth abundance and fairly low cost, use of $\mathrm{Ce}$ would help the commercialization of $n$-type skutterudites for automotive industry only if the thermoelectric properties of Cedoped skutterudites can match those of $\mathrm{Yb}$-doped skutterudites.

Interestingly, single-filled $\mathrm{Ce}-\mathrm{CoSb}_{3}$ skutterudites have been considered as unsuitable thermoelectric materials and there has yet been little report of high temperature thermoelectric properties of pure $\mathrm{Ce}-\mathrm{CoSb}_{3}$ skutterudites. Ce has been considered to be a good auxiliary filler in multiple-filled skutterudites, such as in (In, Ce)-CoSb $\mathrm{Cb}_{3}$ skutterudites ( $z T$ of 1.43 for $\mathrm{In}_{0.2} \mathrm{Ce}_{0.15} \mathrm{Co}_{4} \mathrm{Sb}_{12}$ at $800 \mathrm{~K})^{6}$ or $(\mathrm{Ba}, \mathrm{Ce})-\mathrm{CoSb}_{3}$ skutterudites $(z T$ of 1.26 for $\mathrm{Ba}_{0.18} \mathrm{Ce}_{0.05} \mathrm{Co}_{4} \mathrm{Sb}_{12.02}$ at $\left.850 \mathrm{~K}\right)^{7}$. Ce has not typically been considered as a single filler. This is most likely due to its reported small filling fraction limit $x=0.09$ in $\mathrm{Ce}_{x} \mathrm{Co}_{4} \mathrm{Sb}_{12}$ (ref. 8) that would prevent Ce-containing skutterudites to achieve the optimum carrier concentration for $n$-type skutterudites ${ }^{9}$. To increase the filling fraction limit of Ce in $\mathrm{CoSb}_{3}$ skutterudites, charge-compensational doping, such as $\mathrm{Fe}$ or $\mathrm{Mn}$-doping has been largely applied, which can dope the material from $n$-type to $p$-type ${ }^{10-14}$.

In this study, single-filling $\mathrm{Ce}-\mathrm{CoSb}_{3}$ skutterudites without charge-compensational doping are prepared under equilibrium conditions and their thermoelectric properties are measured up to $850 \mathrm{~K}$. The filling fraction limit of Ce is studied by solubility design using an equilibrium phase diagram approach. This approach introduces both the annealing temperature and nominal composition as variables in determining the filling fraction limit, which leads to the possibility of enhancing filling fraction of fillers thermodynamically rather than consider it as a single value. Using this phase diagram approach, a temperature- dependent solubility like the $\mathrm{Yb}$ in $\mathrm{Yb}-\mathrm{CoSb}_{3}$ sheds light on optimizing $\mathrm{Ce}-\mathrm{CoSb}_{3}$, whose thermoelectric properties are limited by its low filling fraction limit ${ }^{2}$. With solubility design, a clear solubility enhancement with a filling fraction limit of $x=0.20$ is discovered at $850 \mathrm{~K}$ for $\mathrm{Ce}-\mathrm{CoSb}_{3}$ skutterudites. The surprisingly high filling fraction limit at high annealing temperatures enables the Ce-CoSb $b_{3}$ skutterudites to be optimized with $z T$ of 1.3 for $\mathrm{Ce}_{0.14} \mathrm{Co}_{4} \mathrm{Sb}_{12}$ at $850 \mathrm{~K}$, which makes it an excellent substitute for $\mathrm{Yb}$-doped skutterudites for waste heat recovery applications.

\section{Results}

Optimum doping of Ce-CoSb$b_{3}$ skutterudites. Unfilled $\mathrm{CoSb}_{3}$ is an intrinsic semiconductor with carrier concentration $<10^{18} \mathrm{~cm}^{-3}$. Rare earth filler atoms in the void sites are nearly perfect $n$-type donors. The $n$-type carrier concentration is essentially determined by the number of filling atoms and the ionic charge state. In $\mathrm{CoSb}_{3}$ a $\mathrm{Yb}$ filler has a valence charge +2 while $\mathrm{Ce}$ is +3 , which is a lower oxidation state than often observed in oxides but common for Sb compounds ${ }^{15}$. Figure 1 demonstrates the trend of the carrier concentration versus the number of electrons per primitive cell, for either single, double or triple-filled skutterudites. To achieve high power factor of $n$-type skutterudites 0.4-0.6 electrons per $\mathrm{Co}_{4} \mathrm{Sb}_{12}$ unit is required, as indicated by the red-double arrow ${ }^{16}$. The carrier concentration of $\mathrm{Ce}-\mathrm{CoSb}_{3}$ samples in the previous literature study ${ }^{8}$ is limited by the reported low filling fraction limit $\left(x=0.09\right.$ in $\mathrm{Ce}_{x} \mathrm{Co}_{4} \mathrm{Sb}_{12}$ at $973 \mathrm{~K})$, consequently these samples are far from the high-power factor region. To optimize the thermoelectric properties of $\mathrm{Ce}$ single-filled $\mathrm{CoSb}_{3}$ skutterudites, higher filling fraction of Ce is needed. As we shall show later, the filling fraction limit of Ce can be as high as $x=0.20$ at $1,123 \mathrm{~K}$ through solubility design, which is not only more than twice the reported value $x=0.09$ but now allows for the optimization of $\mathrm{Ce}-\mathrm{CoSb}_{3}$. After optimization, the four samples in this work fall in the high power factor region

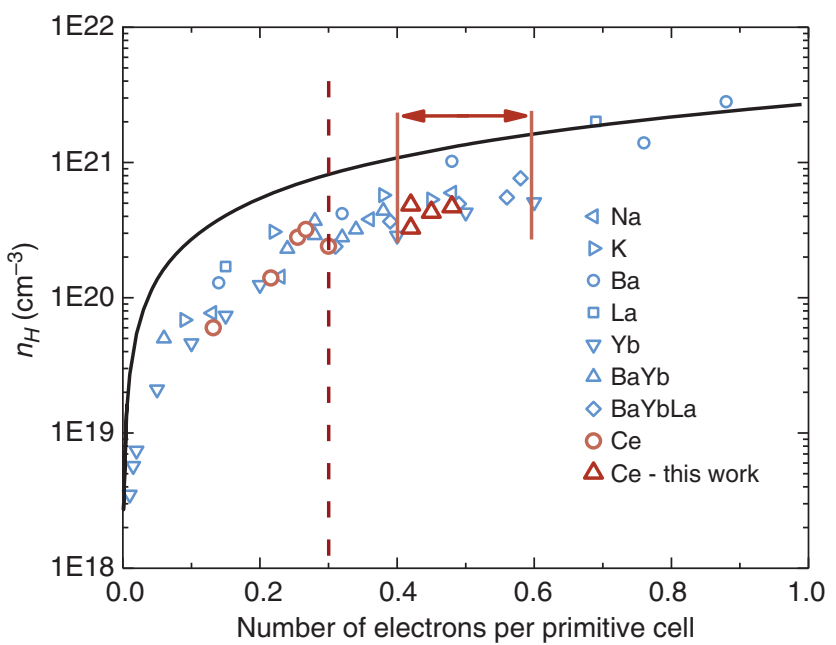

Figure 1 | Hall carrier concentration versus number of electrons per primitive cell $\mathbf{C o}_{\mathbf{4}} \mathbf{S} \mathbf{b}_{\mathbf{1 2}}$. Data from refs $2,8,9,18,19,32-35$. The number of electrons per primitive cell is calculated as the sum of $q_{i}{ }^{*} x_{i}$ for partially filled $\left(R_{1}\right)_{x 1}\left(R_{2}\right)_{x 2} \ldots\left(R_{n}\right)_{x n} \mathrm{Co}_{4} \mathrm{Sb}_{12}$, where $q_{\mathrm{i}}$ is the effective charge state and $x_{\mathrm{i}}$ is the filling fraction of the ith filler $R$ in multiple-filled skutterudites. The solid line represents the theoretical curve calculated by using $n=2 x / a^{3}$, where $x$ is the total number of electrons per primitive cell and $a$ is the lattice constant of $\mathrm{CoSb}_{3}$. The red double arrow represents the estimated high power factor region (see discussions below) and the red dashed line represents the number of electrons per primitive cell limited by the previously thought Ce filling fraction limit 8 . 
(nominal Ce content, $x=0.17,0.18,0.20,0.21$ and actual Ce content measured on hot pressed pellets are $x=0.14,0.14,0.16$, 0.15 , respectively) and all of them have $z T$ values reaching higher than 1.0 and above $750 \mathrm{~K}$ (see Fig. 2e).

Thermoelectric properties of optimized Ce-CoSb 3 skutterudite. The thermoelectric properties can be optimized when the filling fraction $x$ of filler $\mathrm{R}$ (with valence $q$ ) in $\mathrm{R}_{x} \mathrm{Co}_{4} \mathrm{Sb}_{12}$ satisfies $q x=0.4 \sim 0.6$ (refs 9,16). From Fig. 3 we can see that when nominal compositions are in the Ce-rich three-phase regions with an annealing temperature at 1,073 and $1,123 \mathrm{~K}$, the Ce actual content in skutterudite phase is about $x=0.17$ and 0.20 , respectively. With Ce valence state being $+3, q x$ for these materials would be in the optimum region. Thus, a set of samples with nominal compositions $\mathrm{Ce}_{x} \mathrm{Co}_{4} \mathrm{Sb}_{12}(x=0.17,0.18,0.20$ and 0.21$)$ were prepared for thermoelectric evaluation, of which the first two samples were annealed at $1,073 \mathrm{~K}$ and the latter two were annealed at $1,123 \mathrm{~K}$. The temperature dependence of transport properties of these samples are shown in Fig. 2. Moreover, the transport data of two $\mathrm{Yb}$-doped skutterudites with doping level $x=0.20$ and 0.30 are also plotted for the reason of comparison ${ }^{2}$ (Tang, Y. et al. Convergence of multivalley bands as electronic origin of high thermoelectric performance in $\mathrm{CoSb}_{3}$ skutterudites, submitted). With $\mathrm{Yb}$ valence state being $+2, q x$ for these two samples are right on the boundary of the optimum region.

All the samples show negative Seebeck coefficients throughout the whole temperature range, indicating the expected $n$-type semiconductor behaviour. With increasing temperature, both the electrical resistivity and the magnitude of the Seebeck coefficient increase, which is typical behaviour for heavily doped semiconductors. It has to be noted that for the Ce-doped samples, the values of electrical resistivity, seebeck coefficient and thermal conductivity all fall between the values of the two $\mathrm{Yb}$-doped samples, which is in agreement with Fig. $1 . \kappa_{L}$ is obtained by subtracting the electronic contribution from the total thermal conductivity using the Wiedemann-Franz law. We adopted a Lorentz number of $2.0 \times 10^{-8} \mathrm{~V}^{-2} \mathrm{~K}^{-2}$ to be consistent with previous work on skutterudites, and the lattice thermal conductivity calculated accordingly has $<5 \%$ difference from the value
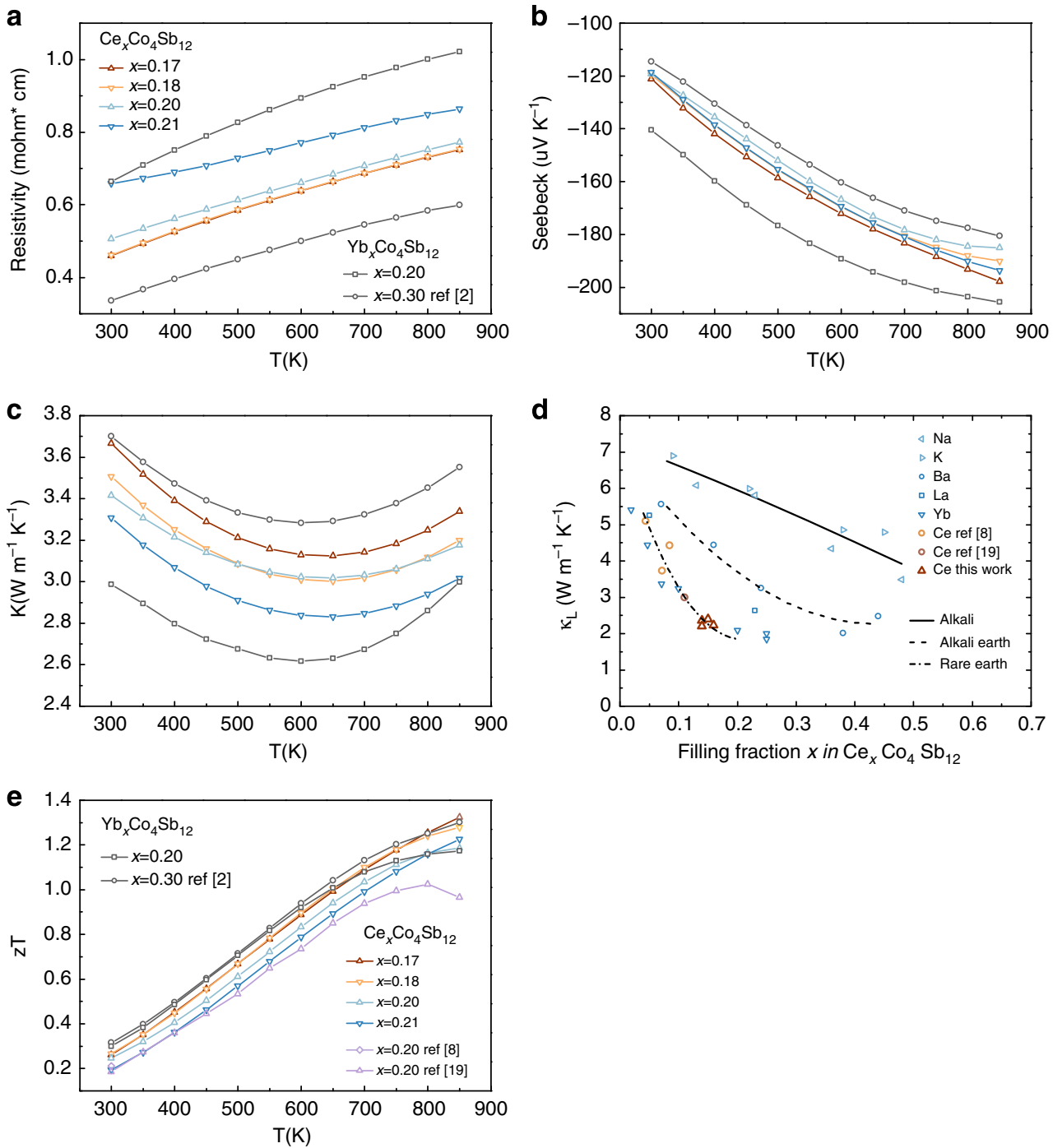

Figure 2 | Transport properties of $\mathbf{C e}$ - and Yb-doped skutterudites. The temperature dependence of: (a) electrical resistivity, (b) seebeck coefficient, (c) thermal conductivity, (e) thermoelectric figure of merit $(z T)$ are plotted in the temperature range of 300-850 K. In figure (d) the lattice thermal conductivity with a Lorentz number of $2.0 \times 10^{-8} \mathrm{~V}^{-2} \mathrm{~K}^{-2}$ is plotted against the filling fraction for various type of fillers. $x$ denotes the nominal doping level of fillers. $\mathrm{Yb}_{\mathrm{x}} \mathrm{Co}_{4} \mathrm{Sb}_{12}$ data for $x=0.20$ is from Tang, $\mathrm{Y}$. et al. Convergence of multivalley bands as electronic origin of high thermoelectric performance in $\mathrm{CoSb}_{3}$ skutterudites, submitted. 


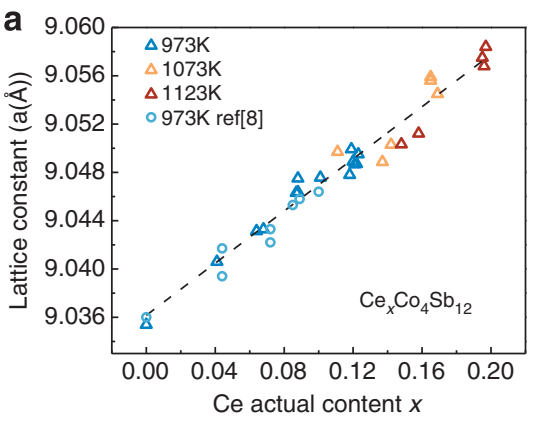

C

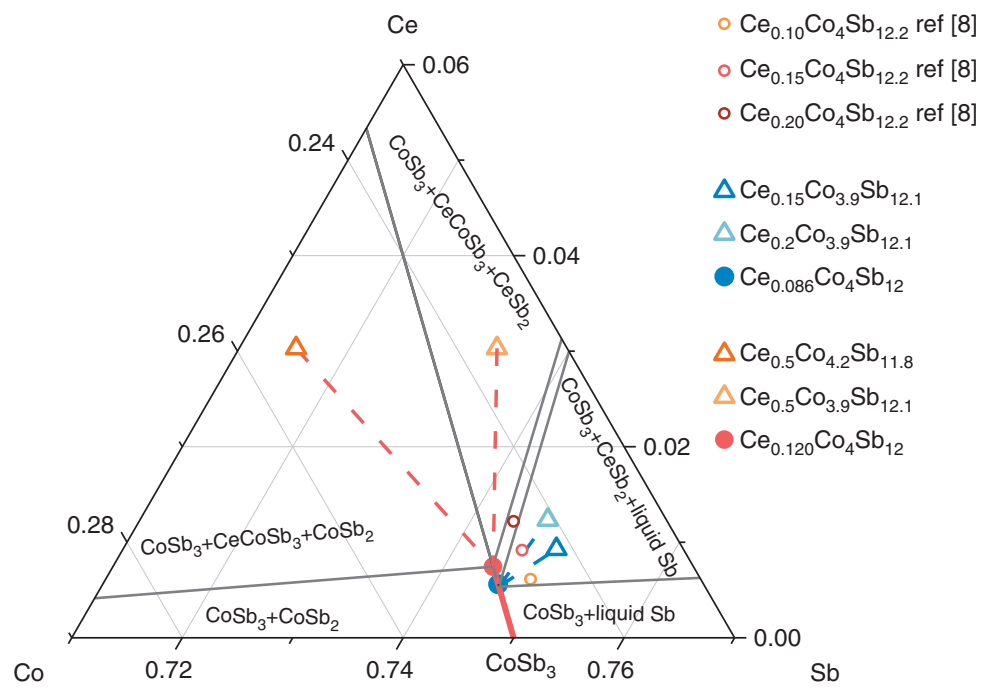

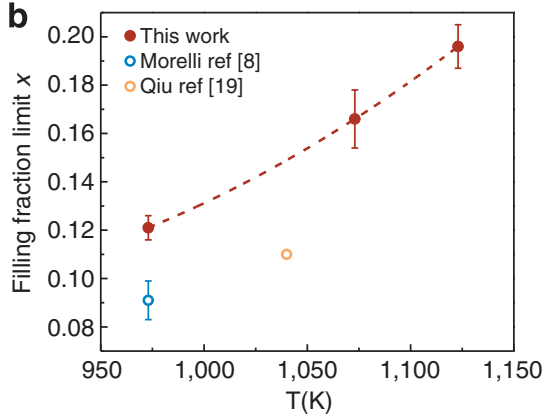

- $\mathrm{Ce}_{0.10} \mathrm{Co}_{4} \mathrm{Sb}_{12.2}$ ref [8]

- $\mathrm{Ce}_{0.15} \mathrm{Co}_{4} \mathrm{Sb}_{12.2}$ ref [8]

- $\mathrm{Ce}_{0.20} \mathrm{Co}_{4} \mathrm{Sb}_{12.2}$ ref [8]

$\triangle \mathrm{Ce}_{0.15} \mathrm{Co}_{3.9} \mathrm{Sb}_{12.1}$

$\triangle \mathrm{Ce}_{0.2} \mathrm{Co}_{3.9} \mathrm{Sb}_{12.1}$

$\mathrm{Sb}$

Figure 3 | Filling fraction limit (FFL) of Ce in Ce-CoSb $\mathbf{3}$ skutterudites. (a) Skutterudite lattice expansion due to Ce filling. (b) Dependence of FFL on annealing temperature. Red dashed line is a guide for the eye. Error bars represent the s.d. of filling fraction limit determined from EPMA measurements. (c) Dependence of FFL on nominal composition with annealing temperature $973 \mathrm{~K}$.

calculated with the Lorentz number determined from a single parabolic band model ${ }^{9,17}$. All Ce-doped samples in this work show strongly reduced $\kappa_{L}$ as compared with binary $\mathrm{CoSb}_{3}$ with $\kappa_{L}$ $\sim 10 \mathrm{Wm}^{-1} \mathrm{~K}^{-1}$ at $300 \mathrm{~K}$. As apparent from Fig. $2 \mathrm{~d}, \mathrm{Ce}$ is as effective in reducing lattice thermal conductivity as $\mathrm{Yb}$, with both much more effective than alkali and alkaline earth element fillers.

Figure 2e shows the continuously increasing $z T$ indicative of the higher doping level than ref. 18. All Ce-CoSb${ }_{3}$ samples in this work have $z T$ values higher than 1.0 at and above $750 \mathrm{~K}$, with the maximum $z T$ value in Ce-doped skutterudite samples reaching 1.3 with nominal Ce content $x=0.17$ and 0.18 at $850 \mathrm{~K}$ (with composition $\mathrm{Ce}_{0.14} \mathrm{Co}_{4} \mathrm{Sb}_{12}$ for both samples determined from Electron Probe Micro-Analysis (EPMA)), which is 30\% higher than that of previously reported literature value for Ce singlefilled skutterudites $\left(z T=1.0 \text { for } \mathrm{Ce}_{0.11} \mathrm{Co}_{4} \mathrm{Sb}_{12} \text { at } 850 \mathrm{~K}\right)^{18}$. These $z T$ are similar to those for single-filled skutterudites ${ }^{2,19-21}$, suggesting that $\mathrm{Ce}$ is a suitable earth abundant and low-cost replacement for other types of fillers, such as $\mathrm{Yb}$, which makes the Ce single-filled skutterudites a more promising candidate for large-scale commercialization. The success of solubility design in $\mathrm{Ce}-\mathrm{CoSb}_{3}$ system is a good example that this is an effective strategy in the optimization of thermoelectric properties in any other ternary systems with filling elements, which allows wide applicability of this strategy.

Ultra-high filling fraction limit of $\mathrm{Ce}$ in $\mathrm{Ce}-\mathrm{CoSb}_{3}$ skutterudites. The lattice expansion due to filling is an easy and effective way of characterizing the amount of fillers actually going into the void site in the skutterudite cell. Here lattice constants were derived from powder X-ray diffraction data and the actual Ce content was determined from EPMA. The lattice constant shows a simple linear dependence on the actual Ce content $x$ in $\mathrm{Ce}_{x} \mathrm{Co}_{4} \mathrm{Sb}_{12}$, which agrees with the literature data and is consistent with $\mathrm{Ce}$ going into the same site across the whole range (up to $x=0.20$ ), presumably the void site. Samples annealed at higher temperatures show both higher Ce content by EPMA as well as higher lattice constants.

The partial filling of $\mathrm{Ce}$ in the void site increases the entropy due to the increase of disorder of the material, and thus it can be expected that the equilibrium solubility should increase measurably with temperature. While this is known theoretically ${ }^{22}$, the filling fraction limit is often considered to be a temperatureindependent quantity; to date, there has been no report on the temperature dependence of filling fraction limit of Ce, let alone using this to design filler solubility in skutterudites. Here we show that the Ce filling fraction limit (determined from red point in Fig. 3c) increases significantly with temperature (see Fig. $3 b$ and Supplementary Fig. 1). It increases to about $0.197 \pm 0.007$ at $1,123 \mathrm{~K}$ and is more than twice the value reported from ref. 8 $(x=0.09)$. This ultra-high filling fraction limit is verified using atom-probe tomography (APT) and is discussed later. This presents an opportunity for optimizing thermoelectric properties of $\mathrm{Ce}-\mathrm{CoSb}_{3}$ skutterudites. The filling fraction limit $x=0.120$ at $973 \mathrm{~K}$ is also $25 \%$ higher than the reported literature value $x=0.09$, which can be explained due to the presence of separate three-phase regions in the $\mathrm{Ce}-\mathrm{Co}-\mathrm{Sb}$ system with different $\mathrm{Ce}$ solubilities in $\mathrm{Ce}_{x} \mathrm{Co}_{4} \mathrm{Sb}_{12}$. 
Figure $3 \mathrm{c}$ shows a magnified region of an isothermal section at $973 \mathrm{~K}$ near $\mathrm{CoSb}_{3}$ of the Ce-Co-Sb ternary phase diagram. More details about phase diagram study are shown in Supplementary Figs 2-4 and Supplementary Table 1.

There are three two-phase regions and three three-phase regions near $\mathrm{CoSb}_{3}$. According to the phase rule, in any threephase region, there are $\mathrm{F}=\mathrm{C}-\mathrm{P}+0=3-3=0$ degrees of freedom where $\mathrm{C}$ is the number of components, $\mathrm{P}$ the number of phases and the 0 indicates both temperature and pressure are fixed. Thus, the compositions of the three equilibrium phases in a three-phase region are fixed and all the nominal compositions in this three-phase region will produce the same skutterudite composition with the same actual Ce content (marked as red and blue points in Fig. 3c), which are called stable skutterudite compositions in this study. This was confirmed by the EPMA measurements to be $x=0.120 \pm 0.005$ for the red stable composition $\left(\mathrm{Ce}_{0.120} \mathrm{Co}_{4} \mathrm{Sb}_{12}\right)$ and $x=0.086 \pm 0.007$ for the blue stable composition $\left(\mathrm{Ce}_{0.086} \mathrm{Co}_{4} \mathrm{Sb}_{12}\right)$ at $973 \mathrm{~K}$.

When the nominal composition is slightly Co rich (in addition to sufficient $\mathrm{Ce}$, for example, $\mathrm{Ce}_{0.5} \mathrm{Co}_{4.2} \mathrm{Sb}_{11.8}$, red empty triangle) the sample is in the three-phase region between $\mathrm{CoSb}_{2}, \mathrm{Ce}-\mathrm{CoSb}_{3}$ (ref. 23) and $\mathrm{Ce}_{x} \mathrm{Co}_{4} \mathrm{Sb}_{12}$ with $x=0.120 \pm 0.005$ (red point). Also if the nominal composition is slightly $\mathrm{Sb}$ rich (for example, $\mathrm{Ce}_{0.5} \mathrm{Co}_{3.9} \mathrm{Sb}_{12.1}$, yellow empty triangle) the sample is in the threephase region between $\mathrm{CeSb}_{2}, \mathrm{Ce}-\mathrm{CoSb}_{3}$ and $\mathrm{Ce}_{x} \mathrm{Co}_{4} \mathrm{Sb}_{12}$ with, coincidentally, the same $x=0.120 \pm 0.005$ (red point) as found in the cobalt-rich three-phase region. In both cases the equilibrium Ce content in skutterudite phase will reach the same filling fraction limit $x=0.120 \pm 0.005$ at $973 \mathrm{~K}$ (red point) as indicated by tie lines (red-dashed lines). Note that these three-phase regions (one with $\mathrm{CeSb}_{2}, \mathrm{Ce}-\mathrm{CoSb}_{3}$ and $\mathrm{Ce}_{x} \mathrm{Co} 4 \mathrm{Sb} 12$; the other with $\mathrm{CoSb}_{2}, \mathrm{Ce}-\mathrm{CoSb}_{3}$ and $\mathrm{CexCo4Sb12)}$ are different from the previous phase diagram study of Ce-Co-Sb system at $400{ }^{\circ} \mathrm{C}^{23}$. No similar ternary phase such as $\mathrm{Ce}-\mathrm{CoSb}_{3}$ is found in the $\mathrm{Yb}$ $\mathrm{Co}-\mathrm{Sb}$ or In-Co-Sb system, but the $\mathrm{Ga}-\mathrm{Co}-\mathrm{Sb}$ system has a ternary phase $\left(\mathrm{Co}_{3} \mathrm{Ga}_{2} \mathrm{Sb}_{4}\right)$ as well ${ }^{24}$.

However, when the nominal composition is very antimony rich, such as used by Morelli ${ }^{8}$ the samples are in another three-phase region between $\mathrm{Ce}_{x} \mathrm{Co}_{4} \mathrm{Sb}_{12}, \mathrm{CeSb}_{2}$ and liquid $\mathrm{Sb}$ (for example, $\mathrm{Ce}_{0.2} \mathrm{Co}_{3.9} \mathrm{Sb}_{12.1}$ and $\mathrm{Ce}_{0.15} \mathrm{Co}_{3.9} \mathrm{Sb}_{12.1}$, marked as green and blue empty triangles, respectively). Here the $\mathrm{Ce}$ content in the skutterudite phase remains constant with $x=0.087 \pm 0.007$ (blue point) as indicated by tie lines (green-and blue-dashed lines). Note that two nominal compositions $\left(\mathrm{Ce}_{0.10} \mathrm{Co}_{4} \mathrm{Sb}_{12.2}\right.$ and $\mathrm{Ce}_{0.15} \mathrm{Co}_{4} \mathrm{Sb}_{12.2}$ ) from ref. 8 fall into this three-phase region of $\mathrm{Ce}_{x} \mathrm{Co}_{4} \mathrm{Sb}_{12}, \mathrm{CeSb}_{2}$ and liquid $\mathrm{Sb}$ and one $\left(\mathrm{Ce}_{0.20} \mathrm{Co}_{4} \mathrm{Sb}_{12.2}\right)$ falls into the two-phase region of $\mathrm{Ce}_{x} \mathrm{Co}_{4} \mathrm{Sb}_{12}$ and $\mathrm{CeSb}_{2}$. The observance of $\mathrm{CeSb}_{2}$ impurity phase in those samples confirms they are in a different field from that which gives the higher $\mathrm{Ce}$ content. The blue stable composition point coincides with the reported filling fraction limit from ref. 8 , which is about $25 \%$ less of the filling fraction limit reported in this study for the same annealing temperature.

From the analysis of the temperature-dependent filling fraction limit we can also predict that the Ce content in the skutterudite phase can be controlled through the annealing temperature when the nominal composition is in one of the three-phase regions, which has proven to be a practical strategy to optimize thermoelectric performances.

APT measurements on $\mathrm{Ce}_{x} \mathrm{Co}_{4} \mathrm{Sb}_{12}$ skutterudite was also performed to confirm the ultra-high filling fraction of $x=0.20$. APT is particularly effective at chemically and structurally characterizing materials in $3 \mathrm{D}$ on the nanometre length scale. It provides accurate information about atomic concentrations and can identify even the smallest nanoparticles ${ }^{25}$. Small nanoparticles of $\mathrm{Yb}_{2} \mathrm{O}_{3}$ and $\mathrm{InSb}$ were observed in some $\mathrm{Yb}$ and In containing skutterudites, respectively ${ }^{6,26,27}$. Such small particles might go unnoticed in the Scanning Electron Microscopy (SEM) and would contribute to the EPMA signal. Here APT was used to verify the Ce content and homogeneity in the $\mathrm{Ce}_{x} \mathrm{Co}_{4} \mathrm{Sb}_{12}$ majority phase for sample annealed at $1,123 \mathrm{~K}$ with nominal composition $\mathrm{Ce}_{0.5} \mathrm{Co}_{4} \mathrm{Sb}_{12}$. According to EPMA, the Ce content in the majority skutterudite phase should be $x=0.20$ or 1.2 at $\%$. Figure 4 a contains a $3 \mathrm{D}$ reconstruction of the skutterudite phase at and around a grain boundary. As can be seen in Fig. 4b, atomic concentrations were consistent and homogeneous away from the grain boundary. No nano-precipitates of Ce-rich phases were observed. Atomic concentrations were averaged over a $30 \mathrm{~nm}$ diameter cylinder, from 20 to $60 \mathrm{~nm}$ on the scale bar in Fig. $4 \mathrm{a}$, and the Ce content was measured to be $1.4 \pm 0.1$ at $\%$. This agrees with the values obtained by EPMA ( $x=0.20$ corresponding to Ce content of 1.2 at $\%$ ) and supports our conclusion that such a high filling fraction of $\mathrm{Ce}$ is indeed uniformly distributed in the skutterudite phase. A uniform accumulation of $\mathrm{Ce}$ at the grain boundary was also observed, accompanied by a reduction in Co. Such atomic segregation is frequently observed at grain boundaries ${ }^{25}$.
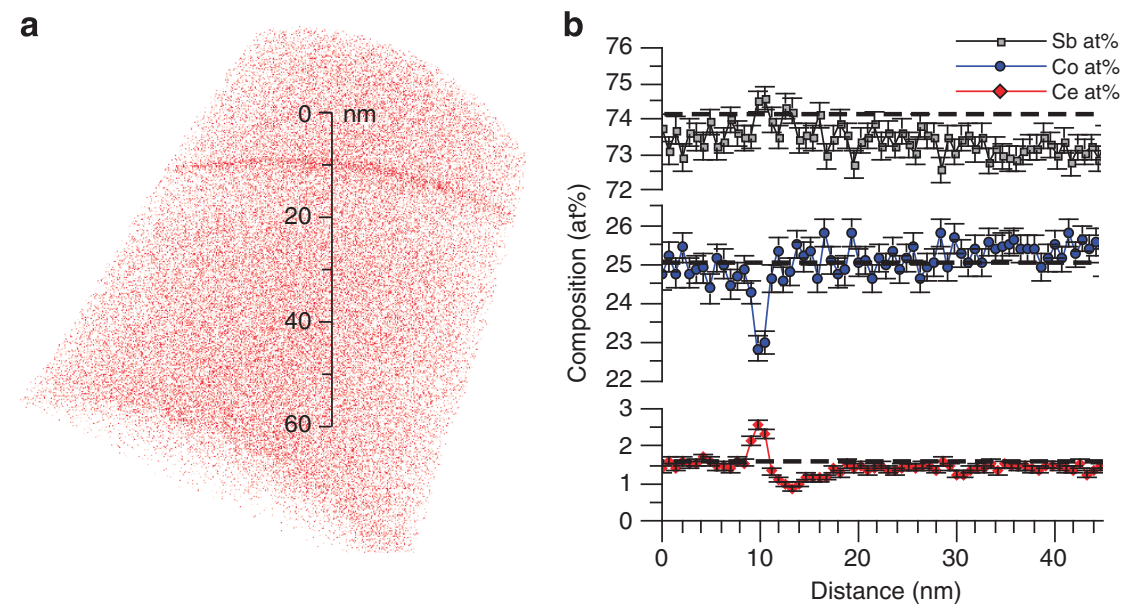

Figure 4 | APT analysis of the most heavily doped sample $\mathbf{C e}_{\mathbf{0 . 2}} \mathbf{C o}_{\mathbf{4}} \mathbf{S b}_{\mathbf{1 2}}$. (a) $3 \mathrm{D}$ reconstruction of microtip containing a grain boundary. Ce atoms are displayed in red; Sb and Co atoms omitted for clarity. (b) Concentration profile across the grain boundary and in the grain. The black dashed lines show values measured by EPMA, and the error bars represent the s.e., $\sqrt{c(1-c) / n}$, where $c$ is the concentration and $n$ is the number of atoms detected in each data point. 


\section{Discussion}

Because of the low cost of $\mathrm{Ce}$ as a single filler, Ce-CoSb 3 skutterudites could have potential for scale-up commercialization in fields such as the automotive industry, if their thermoelectric performance can be optimized. Ce single-filled skutterudites in previous literature studies have been underdoped and fall out of the high power factor region due to the assumed low filling fraction limit of $\mathrm{Ce}(x=0.09)$. Here by conducting an equilibrium phase diagram study, we show that the filling fraction limit has a large temperature dependence and the value can reach as high as $x=0.20$ at $1,123 \mathrm{~K}$. This is more than twice the value reported previously and thus allows to optimize $\mathrm{Ce}-\mathrm{CoSb}_{3}$ skutterudites. This ultra-high Ce content is confirmed by APT measurements. The optimized Ce-CoSb$b_{3}$ skutterudites give a $z T$ value of 1.3 at $850 \mathrm{~K}$ for $\mathrm{Ce}_{0.14} \mathrm{Co}_{4} \mathrm{Sb}_{12}$, which is among the highest reported $z T$ values for single-filled skutterudites.

\section{Methods}

Sample preparation. High-purity elements Co (99.95\%, slug), Sb (99.9999\%, shot) and Ce $(99.9 \%$, rod) purchased from Alfa Aesar were used as raw materials. The samples were sealed in carbon-coated fused silica tubes under vacuum. The silica tubes were heated slowly up to $1,373 \mathrm{~K}$ in $12 \mathrm{~h}$, held at this temperature for $12 \mathrm{~h}$ and then quenched in water to room temperature. Samples were then annealed at temperatures ranging from 973 to $1,123 \mathrm{~K}$ for 7 days. The resulting ingots were hand ground into fine powders and consolidated by rapid hot pressing at $973 \mathrm{~K}$ for $1 \mathrm{~h}$ under a pressure of about $60 \mathrm{MPa}$, yielding fully dense bulk samples. High density ( $>98 \%$ of the theoretical density of $\mathrm{CoSb}_{3}$ ) was achieved in all hot-pressed samples. Hot-pressed samples were sealed in fused tubes under vacuum for further annealing at the same annealing temperatures as before for 7 days again to erase the temperature effect of the hot pressing process before thermoelectric properties were measured ${ }^{2}$. Impurity phases were identified with X-ray diffraction and confirmed with SEM analysis in the same way as described in previous work ${ }^{2,28,29}$.

Thermoelectric property measurements. Electrical transport properties, including electrical conductivity $(\sigma)$ and Seebeck coefficient $(S)$, were measured using the ZEM-3 (ULVAC.) apparatus under a helium atmosphere from 300 to $850 \mathrm{~K}$. Thermal conductivity $(\kappa)$ was calculated using $k=d D_{T} C_{P}$, with the thermal diffusivity $D_{T}$ measured along the cross-plane direction by the laser flash method (Netzsch LFA 457) under argon flow with the Cowan model plus pulse correction. The density of the samples was measured using the geometrical method. The specific heat capacity $C_{P}$ was determined using the Dulong-Petit law $C_{P}=3 k_{B}$ per atom throughout the temperature range $300-850 \mathrm{~K}$. The in-plane Hall coefficient $\left(R_{\mathrm{H}}\right)$ was measured using the Van der Pauw method in a magnetic field up to $2 \mathrm{~T}^{30}$. Hall carrier concentration $(n)$ was then estimated to be equal to $1 / R_{\mathrm{H}} e$, where $e$ is the elementary charge. The Hall carrier mobility $\left(\mu_{\mathrm{n}}\right)$ was calculated according to the relation $\mu_{\mathrm{n}}=R_{\mathrm{H}} \sigma$. The estimated measurement uncertainties are listed as follows: $5 \%$ for electrical resistivity, $7 \%$ for seebeck coefficient, $5 \%$ for thermal diffusivity and $1 \%$ for density. The data precision (reproducibility) is smaller than the accuracy (see Supplementary Fig. 5), leading to $z T$ values within the range of \pm 0.2 .

Atomic probe topographic measurement. APT experiments were conducted on a Cameca LEAP-4000X Si equipped with a picosecond ultaviolet laser (wavelength $355 \mathrm{~nm}$ ). Microtip samples of the nominal composition $\mathrm{Ce}_{0.5} \mathrm{Co}_{4} \mathrm{Sb}_{12}$ were prepared using a dual-beam focused-ion beam microscope (FEI Helios Nanolab) equipped with a micromanipulator (similar to the lift-out method) ${ }^{31}$. Microtips with a diameter of $\sim 100 \mathrm{~nm}$ were fabricated to contain a grain boundary and the last step of the tip sharpening process utilized a low voltage and current $(5 \mathrm{kV}$, $16 \mathrm{pA}) \mathrm{Ga}^{+}$ion beam to minimized $\mathrm{Ga}$ implantation in the sample (Ga content of the region analysed was $<0.01$ at $\%$ ). The sample was maintained at $30 \mathrm{~K}$ and a laser energy of $10 \mathrm{pJ}^{\text {pulse }}{ }^{-1}$ was used at a pulse rate of $250 \mathrm{~Hz}$ with a target evaporation rate of $0.5 \%$ atom pulse ${ }^{-1}$. The primary ions detected were $\mathrm{Co}^{2+}$, $\mathrm{Co}^{+}, \mathrm{Ce}^{3+}, \mathrm{Ce}^{2+}, \mathrm{Sb}^{3+}, \mathrm{Sb}^{2+}$ and $\mathrm{Sb}^{+}$. Very small amounts of $\mathrm{Sb}^{2+}$ and $\mathrm{CoCe}^{+}, \mathrm{Ga}^{+}$and $\mathrm{O}^{2+}$ were also detected. Ions were detected using a twodimensional microchannel plate detector with a detector efficiency of $50 \%$. This detection efficiency is the same for all ions evaporated. The data collected were analysed and a three-dimensional reconstruction was created using the programme IVAS v.3.6.6.

\section{References}

1. Slack, G. A. CRC Handbook of Thermoelectrics 407 (CRC Press, 1995)

2. Tang, Y., Chen, S. W. \& Snyder, G. J. Temperature dependent solubility of yb in $\mathrm{yb}-\cos \mathrm{b}_{3}$ skutterudite and its effect on preparation, optimization and lifetime of thermoelectrics. J. Materiomics 1, 74-83 (2015).
3. Fairbanks, J. W. Vehicular Thermoelectrics: A New Green Technology in Directions in Engine-Efficiency and Emission Research (DEER) Conference Presentations (Detroit, Michigan, 2011).

4. Emsley, J. Nature's Building Blocks: An A-Z Guide to the Elements. New Edn 2011, 120, 614 (Oxford University Press Inc., 2011).

5. The Chinese Rare Earth Website < http://www.zg-xtw.com/> (2015).

6. Li, H., Tang, X., Zhang, Q. \& Uher, C. High performance In[sub x]Ce[sub $y$ ] Co[sub 4] $\mathrm{Sb}$ [sub 12] thermoelectric materials with in situ forming nanostructured InSb phase. Appl. Phys. Lett. 94, 102114 (2009).

7. Bai, S. et al. Enhanced thermoelectric performance of dual-element-filled skutterudites $\mathrm{Ba}_{\mathrm{x}} \mathrm{Ce}_{\mathrm{y}} \mathrm{Co}_{4} \mathrm{Sb}_{12}$. Acta Materialia 57, 3135-3139 (2009).

8. Morelli, D. T., Meisner, G. P., Chen, B., Hu, S. \& Uher, C. Cerium filling and doping of cobalt triantimonide. Phys. Rev. B 56, 7376-7383 (1997)

9. Shi, X. et al. Multiple-filled skutterudites: high thermoelectric figure of merit through separately optimizing electrical and thermal transports. J. Am. Chem. Soc. 133, 7837-7846 (2011).

10. Zhang, L. et al. Thermoelectric performance of mischmetal skutterudites MmyFe4 - xCoxSb12 at elevated temperatures. J. Alloy Compd. 490, 19-25 (2010).

11. Liu, R., Qiu, P., Chen, X., Huang, X. \& Chen, L. Composition optimization of p-type skutterudites CeyFexCo4-xSb12 and YbyFexCo4-xSb12. J. Mater. Res. 26, 1813-1819 (2011).

12. Tang, X., Zhang, Q., Chen, L., Goto, T. \& Hirai, T. Synthesis and thermoelectric properties of p-type- and n-type-filled skutterudite $\mathrm{R}$ [sub y] M[sub x]Co[sub $4-\mathrm{x}] \mathrm{Sb}[$ sub 12](R:Ce,Ba,Y;M:Fe,Ni). J. Appl. Phys. 97, 093712 (2005).

13. Chen, B. et al. Low-temperature transport properties of the filled skutterudites CeFe4-xCoxSb12. Phys. Rev. B 55, 1476-1480 (1997).

14. Meisner, G., Morelli, D., Hu, S., Yang, J. \& Uher, C. Structure and lattice thermal conductivity of fractionally filled skutterudites: solid solutions of fully filled and unfilled end members. Phys. Rev. Lett. 80, 3551-3554 (1998).

15. Uher, C. in Semiconductors and Semimetals. Vol. 69 (ed. Tritt, T. M.) Ch. 5 14-16 (Academic Press, 2000).

16. Yang, J., Xi, L., Zhang, W., Chen, L. D. \& Yang, J. Electrical transport properties of filled cosb3 skutterudites: a theoretical study. J. Electron. Mater. 38, 1397-1401 (2009).

17. Kim, H.-S., Gibbs, Z. M., Tang, Y., Wang, H. \& Snyder, G. J. Characterization of Lorenz number with Seebeck coefficient measurement. APL Mater. 3, 041506 (2015).

18. Qiu, P. et al. Enhancement of thermoelectric performance in slightly charge-compensated CeyCo4Sb12 skutterudites. Appl. Phys. Lett. 103, 062103 (2013).

19. Chen, L. D. et al. Anomalous barium filling fraction and n-type thermoelectric performance of $\mathrm{Ba}$ [sub y] $\mathrm{Co}[$ sub 4] Sb[sub 12]. J. Appl. Phys. 90, 1864 (2001).

20. Yang, J. et al. Solubility study of Yb in n-type skutterudites YbxCo4Sb12 and their enhanced thermoelectric properties. Phys. Rev. B 80, 115329 (2009).

21. Nolas, G. S., Kaeser, M., Littleton, R. T. \& Tritt, T. M. High figure of merit in partially filled ytterbium skutterudite materials. Appl. Phys. Lett. 77, 1855-1857 (2000).

22. Shi, X., Zhang, W., Chen, L. D., Yang, J. \& Uher, C. Thermodynamic analysis of the filling fraction limits for impurities in CoSb3 based on ab initio calculations. Acta Materialia 56, 1733-1740 (2008).

23. Luo, R. M., Liu, F. S., Li, J. Q. \& Feng, X. W. The isothermal section of the $\mathrm{Ce}-\mathrm{Co}-\mathrm{Sb}$ ternary system at $400^{\circ} \mathrm{C}$. J. Alloy Compd. 471, 60-63 (2009).

24. Gierlotka, W. et al. The Co-Sb-Ga system: isoplethal section and thermodynamic modeling. METALL MATER TRA 46, 1488-1499 (2015).

25. Kim, Y.-J. et al. Three-dimensional atom-probe tomographic analyses of leadtelluride based thermoelectric materials. JOM 66, 2288-2297 (2014).

26. Ding, J. et al. Creation of $\mathrm{Yb} 2 \mathrm{O} 3$ nanoprecipitates through an oxidation process in bulk yb-filled skutterudites. J. Electron. Mater. 42, 382-388 (2013).

27. Zhao, X. Y. et al. Synthesis of $\mathrm{Yb}$ [sub y] Co[sub 4] Sb[sub 12]/Yb[sub 2] O[sub 3] composites and their thermoelectric properties. Appl. Phys. Lett. 89, 092121 (2006).

28. Qiu, Y. et al. Charge-compensated compound defects in ga-containing thermoelectric skutterudites. Adv. Funct. Mater. 23, 3194-3203 (2013).

29. Tang, Y. et al. Phase diagram of In-Co-Sb system and thermoelectric properties of In-containing skutterudites. Energy Environ. Sci. 7, 812-819 (2014).

30. Borup, K. A. et al. Measurement of the electrical resistivity and Hall coefficient at high temperatures. Rev. Sci. Instrum. 83, 123902 (2012).

31. Miller, M. K., Russell, K. F., Thompson, K., Alvis, R. \& Larson, D. J. review of atom probe FIB-based specimen preparation methods. Microsc. Microanal. 13, 428-436 (2007).

32. Nolas, G. S., Cohn, J. L. \& Slack, G. A. Effect of partial void filling on the lattice thermal conductivity of skutterudites. Phys. Rev. B. 58, 164-170 (1998).

33. Pei, Y. Z. et al. Synthesis and thermoelectric properties of $\mathrm{K}[\mathrm{sub} \mathrm{y}] \mathrm{Co}[\mathrm{sub}$ 4]Sb[sub 12]. Appl. Phys. Lett. 89, 221107 (2006). 
34. Pei, Y. Z. et al. Improving thermoelectric performance of caged compounds through light-element filling. Appl. Phys. Lett. 95, 042101 (2009).

35. Shi, X. et al. Low thermal conductivity and high thermoelectric figure of merit in n-type $\mathrm{Ba}$ [sub $\mathrm{x}] \mathrm{Yb}[$ sub y] $\mathrm{Co}$ [sub 4] $\mathrm{Sb}$ [sub 12] double-filled skutterudites. Appl. Phys. Lett. 92, 182101 (2008).

\section{Acknowledgements}

We acknowledge the financial support of Solid-State Solar-Thermal Energy Conversion Center (S3TEC), an Energy Frontier Research Center funded by the U.S. Department of Energy, Office of Science, Basic Energy Sciences under Award No. DE-SC0001299. We also acknowledge the financial support of National Science Council of Taiwan (NSC1013113-P-008-001). We thank Yulong Li, Xun Shi, Lidong Chen of Shanghai Institute of Ceramics, Chinese Academy of Sciences for ZEM-3 measurements. This work made use of the EPIC facility (NUANCE Center-Northwestern University), which has received support from the MRSEC program (NSF DMR-1121262) at the Materials Research Center; the Nanoscale Science and Engineering Center (NSF EEC-0647560) at the International Institute for Nanotechnology; and the State of Illinois, through the International Institute for Nanotechnology. The LEAP at the Northwestern University Center for Atom-Probe Tomography (NUCAPT) was acquired and upgraded with equipment grants from the MRI program of the National Science Foundation (grant number DMR0420532) and the DURIP program of the Office of Naval Research (grant numbers N00014-0400798, N00014-0610539, N00014-0910781). NUCAPT is supported by the National Science Foundation's MRSEC program (grant number DMR-1121262). Additional instrumentation at NUCAPT was supported by the Initiative for Sustainability and Energy at Northwestern (ISEN). We acknowledge further support from the International S\&T Cooperation Program of China (2015DFA51050). Thank you to Dieter Isheim for critical conversations regarding experimental set-up and data analysis.

\section{Author contributions}

Y.T. and G.J.S. contributed equally to design the study and write the manuscript. Y.T. contributed to sample synthesis, structure characterization and property measurements. R.H. contributed to APT analysis and manuscript refinement. S.W.C. contributed to the discussion of phase diagram results. Y.T., R.H. and G.J.S. contributed to the thumbnail figure design.

\section{Additional information}

Supplementary Information accompanies this paper at http://www.nature.com/ naturecommunications

Competing financial interests: The authors declare no competing financial interests.

Reprints and permission information is available online at http://npg.nature.com/ reprintsandpermissions/

How to cite this article: Tang, Y. et al. Solubility design leading to high figure of merit in low-cost Ce-CoSb 3 skutterudites. Nat. Commun. 6:7584 doi: 10.1038/ncomms8584 (2015).

\section{(c) (i)}

This work is licensed under a Creative Commons Attribution 4.0 International License. The images or other third party material in this article are included in the article's Creative Commons license, unless indicated otherwise in the credit line; if the material is not included under the Creative Commons license, users will need to obtain permission from the license holder to reproduce the material. To view a copy of this license, visit http://creativecommons.org/ licenses/by/4.0/ 